\title{
Procedure for determining transport and warehousing systems characteristics in industrial enterprises in megapolis
}

\author{
Anton Radaev ${ }^{1, *}$, Valery Leventsov ${ }^{1}$, and Vladimir Kobzev ${ }^{1}$ \\ ${ }^{1}$ Graduate School of Industrial Management and Economics, Peter the Great Saint-Petersburg Polytechnic University, 195251 \\ Saint-Petersburg, Russia
}

\begin{abstract}
The most common problem among a vast variety of design and performance management problems for the industrial enterprises supply chain networks is determining transport and storage processes' characteristics in the areas of high population density. The divergence of the logistic structure development in the area has a major influence on the choice of location for the warehouses and the characteristics of the corresponding material flows. This article addresses the issues of determining transport and warehousing systems characteristics in a major population center and its suburbs. In particular, this means the warehouse location and the delivery lot sizes. The article suggests a procedure for solving this problem based on a set of mathematical models using linear and non-linear optimization via the standard algorithms of common computer software like Microsoft Excel, Mathcad, MatLab, etc.
\end{abstract}

\section{Introduction}

Efficient functioning of the industrial enterprises in modern conditions is impossible without a detailed elaboration on the questions of organization design and performance management. This concerns not only manufacturing systems of said enterprises, but also the corresponding supply chains, which facilitate the material flows with the help of transport and warehousing resources of industrial enterprises and external party contractors. The importance of solving the design and operation problems for industrial enterprises and their supply chains is for the most part determined by the following: the added complexity and growing intensity of the material and assistant flows accompanied by market globalization, and the tightening of the requirements for the products' flow and transformation, which provides competitive ability for the enterprise. Therefore, the parameters of the material flow structure from the supplier to the consumer and the storage buffer locations are considered the most significant characteristics for organization design and performance management of the industrial enterprises' supply chains. It stands to mention that choosing the locations for the storage buffers is especially important for servicing the ultimate consumers in large residential areas, which will be hereafter referred to as megapolises.

This circumstance ascertains the viability of this research. The goal of the research is to develop a procedure for determining transport and warehousing network's characteristics (as a structural unit of a supply chain) for an industrial enterprise in a megapolis environment. The aforementioned procedure should take into account the specific features of logistic processes in megapolises and the surrounding territories. These features include the following:

- products are transferred in delivery lotes in the transport and warehousing network using vehicles of a certain capacity;

- vehicles travel in the complex set of roads, equipped with traffic lights and other means of traffic regulation;

- warehouse upkeep and rent cost (whether rented or owned) is related to its location, which is determined by the specifics of the city infrastructure.

The warehouses' locations and the volumes of transferred goods between the nodes of the supply chain network are considered the most important characteristics of the transport and warehousing network for this research.

It is also worth mentioning that in order to assure feasible results, the proffered procedure should be implemented by the aid of the standard computing algorithms using software like "Microsoft Excel", "Mathcad", "MatLab" etc.

This paper is organized as follows. Section 2 contains the results of the literature review and analysis in the field of determining the supply chain characteristics for enterprises. In section 3 the problem of determining the transport and warehousing network's characteristics in industrial enterprises in a megapolis environment is posed. Section 4 includes the procedure for solving the

\footnotetext{
Corresponding author: TW-inc@yandex.ru
} 
problem. Section 5 provides the results and conclusions of the research and also the direction for future work.

\section{Literature review}

The first stages of the research include the literature review for the field of interest. The results of this review and analysis are presented in the following.

Many of the works are focusing on determining the supply chain characteristics in relation to delivery lot sizes and locations of various supply chain elements. The techniques used are mostly linear and non-linear optimization models. The main objectives are the total operating expenses in the supply chain and some cost indicators derived from those. The suggested solving methods are Lagrangian relaxation-based method [1], genetic algorithms $[2,3,4,5]$, game theory methods [6], heuristic methods $[7,8,9,10]$, decision-making methods $[11,12,13]$, fuzzy optimization [14], ant colony algorithm [15], particle swarm optimization [16], modified fruit fly optimization [17], other linear and non-linear techniques [18, 19, 20, 21].

A range of works concerns the development and implementation of techniques and tools based on Economic Order Quantity and Economic Production Quantity models for determining the characteristics of the supply process. The proposed modifications of the aforementioned models account for a large product range [22], the uncertainty of the demand, delivery lot sizes and the corresponding operation cycles [23, 24, 25], lead times [26], backorders [27], vendor-buyer relationships, in regard to consignment stock among other things [28]. The proposed solving methods are numerical methods [25], genetic algorythms [22] and game theory methods [28].

The analysis of the works mentioned above prompted the following conclusions:

- the aforecited works treat transport processes in a simplified, amplified way and they do not account for the specifics of the corresponding process (the arrangement of goods in the cargo area, the traffic in an urban environment), technical features of the vehicle (active storage of the truck body, carrying capacity, mean velocity on the route etc.) and transportation rates (the transportation cost proportional to distance or duration of the traffic route - for intraregional and transregional shipments);

- the suggested mathematical models do not account for the expenses concerning upkeep or rent of the fixed assets in the supply chain structure; for instance, the rent cost per stacking capacity unit of a distribution warehouse for serving the customers in a megapolis will vary considerably depending on the location of a warehouse in said megapolis.

In conclusion, the aforementioned studies do not make allowance for a wide range of specific ways to organize logistic processes in the areas with high population density. Therefore, these studies can only be effective for the approximate, rough design of the supply process of the industrial enterprises. This circumstance serves to prove the high relevance and applicability of the conducted research.

\section{Problem definition}

This section of the article contains the generalized problem definition for determining the transport and warehousing system's characteristics in industrial enterprises in a megapolis environment. It concerns the main principles of the procedure, the input data and the decision variables.

\subsection{Main principles}

- The main object of the research is a transport and warehousing network. This network is an element of a supply chain of an industrial enterprise for which the products are transferred in a certain geographical area from a number of suppliers to a number of consumers through an only distribution warehouse where the products are temporarily stored.

- The goods are essentially put in standardized packages assembled on flat pallets.

- The goods are stored in a rented warehouse in a certain geographical area, which includes a megapolis and its suburbs. Given that, there is a functional relation between the warehouse characteristics (storage area, active height and cost per stacking capacity unit of the warehouse) and the warehouse location specifics. For this research, it will be practical to suggest that the location of a warehouse is determined by the shortest distance between the warehouse and the nominal center of the megapolis. The center is a location where the aforementioned characteristics of a warehouse reach extreme values $[29,30]$. This point is illustrated by fig. 1 .

- The goods are transferred from each supplier to the distribution warehouse and from there to each consumer with the aid of road transport with certain technical features (carrying capacity, velocity etc.) and certain costs. In case of intraregional shipments the costs can be divided into fixed (related to serving the vehicle) and variable costs (determining the monetary equivalent of 1 hour's work of a vehicle).

- The goods are transferred from each supplier to the warehouse in fixed volumes in regular intervals of time equivalent to the preferable supply interval. The goods are transferred from the distribution warehouse to the ultimate consumers daily and in fixed volumes.

- It is required to determine the optimal location of the warehouse and the optimal delivery lot sizes for the shipments from the suppliers to the warehouse. These parameters should ensure the minimal total expenses for warehouse upkeep and rent and for transportation within the confines of the examined transport and warehousing network. 


\subsection{Input data}

$Z$ - number of items on stock in the transport and warehousing network; $m, n$ - accordingly, the number of suppliers and consumers in the examined geographic area;

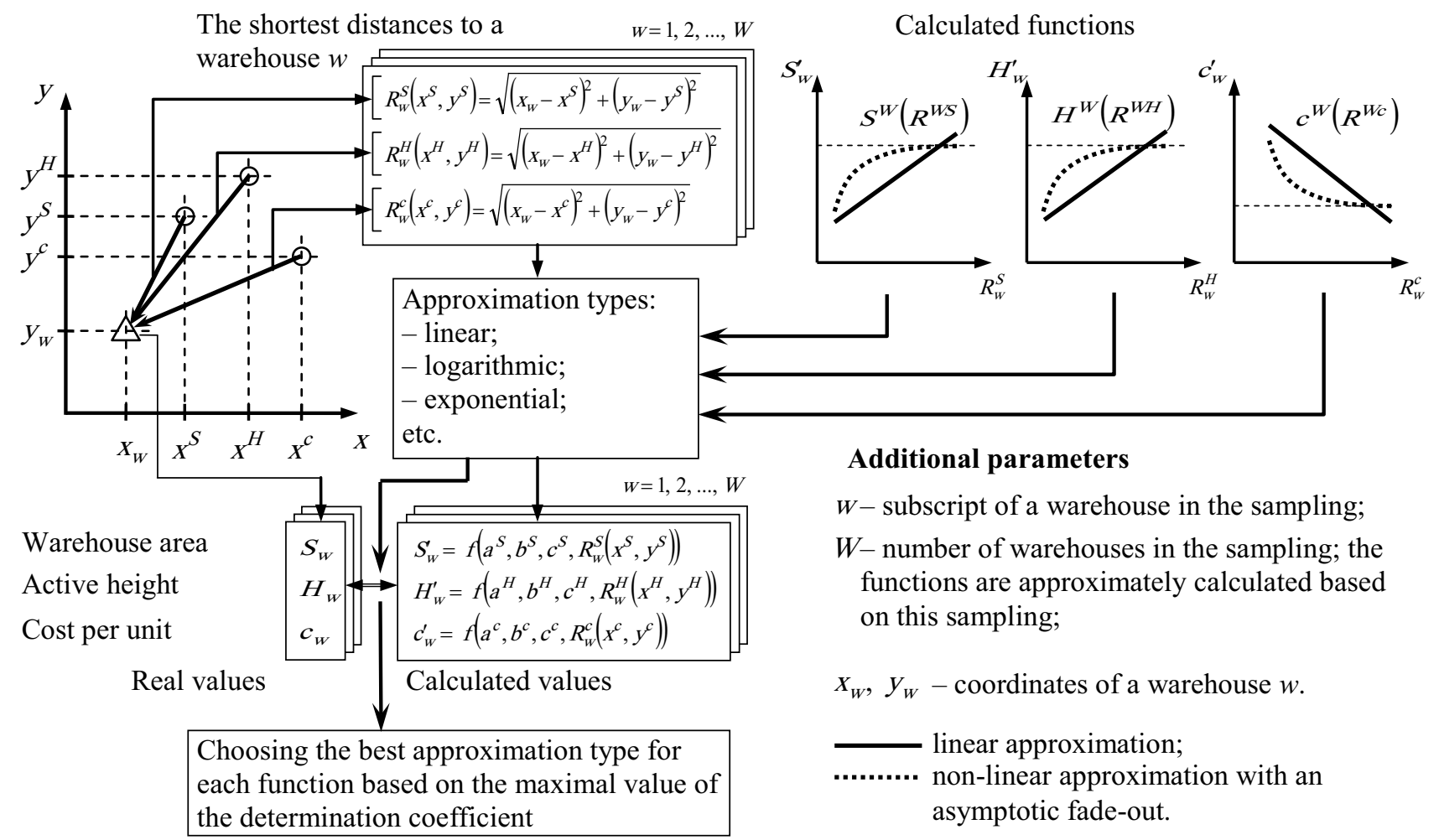

Fig. 1. Description of the calculated functions for the warehouse characteristics in relation to its location, and the procedure for determining the functions.

$k=1, \ldots, z-$ subscripts of stock items;

$i=1, \ldots, m-$ subscripts of suppliers;

$j=1, \ldots, n-$ subscripts of consumers;

$L^{U}, B^{U}, H^{U}$ - accordingly, reference values (m) for the length, width and height of a cargo unit for the store item;

$x_{i(j)}, y_{i(j)}$ - location coordinates $(\mathrm{km})$ of supplier $i$ (consumer $j$ ) in the Cartesian system, based on a map of the examined geographical area;

$R_{i(j)}$ - preferable supply interval (workdays) for supplier $i$ to the distribution warehouse (from the warehouse to consumer $j$ ); $R_{j}=1$ workday;

$Q_{k i}^{\max }$ - maximal delivery lot size (pcs/shipment) for stock item $k$ for supplier $i$;

$I_{k j}$ - expected daily volume of shipments (pcs/ shipment) for stock item $k$ from the warehouse to consumer $j$;

$r_{i(j)}$ - active storage (pcs/unit) of the truck body for the vehicles used by supplier $i$ (consumer $j$ ) - calculated based on reference values of mass and dimensional parameters of the truck body and cargo units, which can be evaluated by known formulas [29];

$v_{i(j)}$ - mean velocity on the route $(\mathrm{km} / \mathrm{hr})$ of the vehicles used by supplier $i$ (consumer $j$ ) [29];
$c_{i(j)}^{T 0}, \quad c_{i(j)}^{T 1}-$ accordingly, fixed (c.u../run) and variable (c.u./(unit.hr)) costs for the vehicles used by supplier $i$ (consumer $j$ );

$x^{\min (\max )}, y^{\min (\max )}-\operatorname{minimal}$ (maximal) possible values for the abscess and ordinate coordinates accordingly;

$k^{T I}$ - coefficient accounting for the specifics of the transport infrastructure [29];

$k_{i(j)}^{T L}$ - coefficient accounting for standing time at the traffic lights for the vehicles used by supplier $i$ (consumer $j$ ), - it is determined based on the mean velocity on the route $v_{i(j)}$ using a known formula [29];

$\Delta_{h}$ - vertical distance between the storage units, which is defined by the storage technique, e.g. the design of the rack;

$k^{S}$ - space occupancy coefficient for the warehouse, which is defined by the storage technique; e.g. for rack storage $k^{S}=0.39 \div 0.45$;

$T$ - length (workdays) of the given time period;

$S^{W}\left(R^{W S}\right), \quad H^{W}\left(R^{W H}\right), \quad c^{W}\left(R^{W c}\right)$ - functions for determining the warehouse area $S^{W}\left(\mathrm{~m}^{2}\right)$, active height $H^{W}$ (m) and rent cost per stacking capacity unit of the warehouse $c^{W}$ (c.u./(unit.pd)) in relation to the distance 
from the center $R^{W S(H, c)}(\mathrm{km})$. Each function is defined based on the warehouse sampling in the examined geographical area (the parameters, such as location, area, active height and cost per unit are known). This is accomplished by implementing the following stages (fig. 1): creating the alternative approximations (linear, logarithmic, exponential etc.); optimization of the approximating function coefficients $a^{S(H, c)}, b^{S(H, c)}$, $c^{S(H, c)}$ and center coordinates $x^{S(H, c)}, y^{S(H, c)}$ using the generalized reduced gradient method; choosing the best approximation based on the maximal value of the determination coefficient. The procedure for determining the approximation coefficients concerning the warehousing system parameters is described in [30]; $x^{S(H, c)}, y^{S(H, c)}-$ coordinates $(\mathrm{km})$ of the nominal center used to calculate the area of the warehouse (its height and cost per unit).
$Q_{k i}$ - delivery lot volume (pcs/shipment) for stock item $k$ for supplier $i$;

$x^{W}, \quad y^{W}-$ coordinates $(\mathrm{km})$ of the distribution warehouse in the Cartesian system, based on a map of the examined geographical area.

\section{Solving procedure}

The procedure for determining the transport and warehousing system characteristics in industrial enterprises in a megapolis environment is defined by the succession of certain stages. The detailed description will be given further.

The first stage for implementing the procedure is to compose the calculated parameters for the optimization models which will be used in the next stages. The mentioned parameters are defined in Table 1.

\subsection{Decision variables}

Table 1. Calculated parameters, units of measure and equations.

\begin{tabular}{|c|c|c|c|}
\hline No. & Parameter & Unit & Equation \\
\hline 1 & \multicolumn{3}{|c|}{ Stock item $k$, supplied by supplier $i$} \\
\hline 1.1 & Volume of the daily demand & \begin{tabular}{c|} 
pcs/ \\
workdays
\end{tabular} & $=\frac{Q_{k i}}{R_{i}}$ \\
\hline 2 & \multicolumn{3}{|r|}{ Supplier $i$, consumer $j$} \\
\hline 2.1 & Shortest distance to the warehouse & $\mathrm{km}$ & $L_{i(j)}^{W}\left(x^{W}, y^{W}\right)=\sqrt{\left(x_{i(j)}-x^{W}\right)^{2}+\left(y_{i(j)}-y^{W}\right)^{2}}$ \\
\hline 2.2 & $\begin{array}{l}\text { Transportation cost for one run to the } \\
\text { warehouse (and from the warehouse) }\end{array}$ & c.u./ run & $C_{i(j)}^{T}\left(x^{W}, y^{W}\right)=c_{i(j)}^{T 0}+c_{i(j)}^{T 1} \cdot \frac{L_{i(j)}^{W}\left(x^{W}, y^{W}\right) \cdot k^{T I} \cdot k_{i(j)}^{T L}}{v_{i(j)}}$ \\
\hline 2.3 & Cargo volume in a shipment & $\begin{array}{c}\text { pcs/ } \\
\text { shipment }\end{array}$ & $Q_{i}\left(\left\{Q_{k i}\right\}\right)=\sum_{k=1}^{Z} Q_{k i} ; \quad Q_{j}=\sum_{k=1}^{Z} I_{k j}$ \\
\hline 2.4 & $\begin{array}{l}\text { Mean number of runs in the given } \\
\text { time period }\end{array}$ & ea/ pd & $z_{i}^{T}\left(\left\{Q_{k i}\right\}\right)=\frac{Q_{i}\left(\left\{Q_{k i}\right\}\right)}{r_{i}} \cdot \frac{T}{R_{i}} ; \quad z_{j}^{T}=\frac{Q_{j}}{r_{j}} \cdot \frac{T}{R_{j}}$ \\
\hline 3 & \multicolumn{3}{|c|}{ Transport and warehousing network } \\
\hline 3.1 & Needed warehouse capacity & pcs & $\sum_{i=1}^{m} Q_{i}\left(\left\{Q_{k i}\right\}\right)$ \\
\hline 3.2 & $\begin{array}{c}\text { Distance from the nominal center to } \\
\text { the warehouse used to calculate the } \\
\text { area of the warehouse (its height and } \\
\text { cost per unit) }\end{array}$ & $\mathrm{km}$ & $\left.H, c)-x^{W}\right)^{2}+\left(y^{S(H, c)}-y^{W}\right)^{2}$ \\
\hline 3.3 & Expected warehouse capacity & pes & $V^{W}\left(x^{W}, y^{W}\right)=\left\lfloor\frac{S^{W}\left(R^{W S}\left(x^{W}, y^{W}\right)\right) \cdot k^{S}}{L^{U} \cdot B^{U}}\right\rfloor \cdot\left\lfloor\frac{H^{W}\left(R^{W H}\left(x^{W}, y^{W}\right)\right)}{H^{U}+\Delta_{h}}\right\rfloor$ \\
\hline 3.4 & $\begin{array}{l}\text { Warehouse rent cost in the given time } \\
\text { period }\end{array}$ & c.u./pd & $C_{\Sigma}^{W}\left(x^{W}, y^{W}\right)=c^{W}\left(R^{W c}\left(x^{W}, y^{W}\right)\right) \cdot V^{W}\left(x^{W}, y^{W}\right)$ \\
\hline 3.5 & $\begin{array}{l}\text { Assumed storage cost for the supply } \\
\text { stock in the given time period }\end{array}$ & c.u./pd & $\widetilde{C}_{\Sigma}^{W}\left(\left\{Q_{k i}\right\}\right)=c^{W}\left(R^{W c}\left(x^{W}, y^{W}\right)\right) \cdot \bar{V}^{W}\left(\left\{Q_{k i}\right\}\right)$ \\
\hline 3.6 & $\begin{array}{c}\text { Total transportation cost from } \\
\text { suppliers to the warehouse in the given } \\
\text { time period }\end{array}$ & c.u./pd & $C_{\Sigma}^{T i n}\left(\left\{Q_{k i}\right\}, x^{W}, y^{W}\right)=\sum_{i=1}^{m} C_{i}^{T}\left(x^{W}, y^{W}\right) \cdot z_{i}^{T}\left(\left\{Q_{k i}\right\}\right)$ \\
\hline 3.7 & $\begin{array}{c}\text { Total transportation cost from the } \\
\text { warehouse to consumers in the given } \\
\text { time period }\end{array}$ & c.u./pd & $C_{\Sigma}^{T \text { out }}\left(x^{W}, y^{W}\right)=\sum_{i=1}^{m} C_{i}^{T}\left(x^{W}, y^{W}\right) \cdot z_{j}^{T}$ \\
\hline
\end{tabular}




\begin{tabular}{|c|c|c|c|}
\hline 3.8 & $\begin{array}{c}\text { Total transportation cost in the given } \\
\text { time period }\end{array}$ & c.u./pd & $C_{\Sigma}^{T}\left(\left\{Q_{k i}\right\}, x^{W}, y^{W}\right)=C_{\Sigma}^{T \text { in }}\left(\left\{Q_{k i}\right\}, x^{W}, y^{W}\right)+C_{\Sigma}^{T o u t}\left(x^{W}, y^{W}\right)$ \\
\hline 3.9 & $\begin{array}{c}\text { Total rent and transportation cost in } \\
\text { the given time period }\end{array}$ & c.u./pd & $C_{\Sigma}\left(\left\{Q_{k i}\right\}, x^{W}, y^{W}\right)=C_{\Sigma}^{W}\left(x^{W}, y^{W}\right)+C_{\Sigma}^{T}\left(\left\{Q_{k i}\right\}, x^{W}, y^{W}\right)$ \\
\hline
\end{tabular}

Note: $[\ldots]$-means the result of rounding down the value to a nearest whole number.

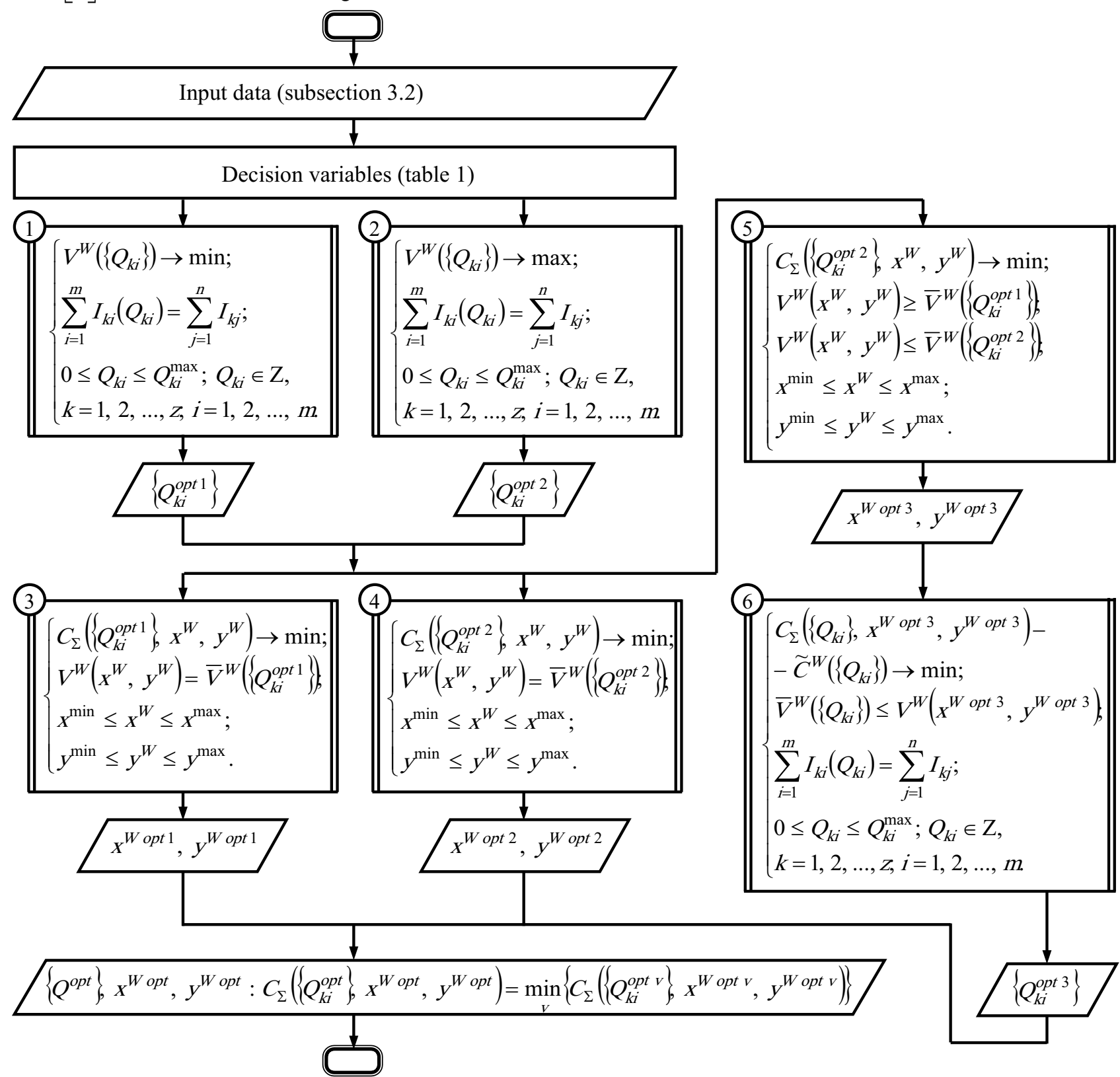

Fig. 2. Calculated functions for the warehouse's characteristics in relation to its location, and the procedure for determining said functions.

The next stages of the procedure are best described by the flow chart in fig. 2 . It seems to be impossible to calculate all the decision variables for the given problem by a single optimization model and its implementation via the standard methods, because there are too many miscellaneous variables. Therefore, the procedure suggests a consequent (step-by-step) optimization for groups of variables using certain guidelines.

The third stage of the procedure shows the implementation of two optimization models for determining the delivery lot volumes with minimal (the goods are transferred to the warehouse at a fast rate in small delivery lots) and maximal (the goods are transferred rarely and in large delivery lots) needed warehouse capacity (boxes 1 and 2 in fig. 2).

The fourth stage of the procedure describes the optimization of the location of the warehouse of a minimal and maximal capacity (boxes 3 and 4 in fig. 2). It is based on the optimal (see stage 3) delivery lot volumes. Thus, upon implementing this stage the first two alternate solutions for the problem (sets of optimal values of the decision variables) are derived.

The fifth stage is used to create the last alternative solution for the problem. To begin with, the optimal location of the warehouse is determined using the total cost minimization criteria (box 5 in fig.2). The total cost 
is related to the delivery lot size, which is in turn determined by maximizing the warehouse capacity (box 2 in fig.2). At that, the expected warehouse capacity is restricted by the threshold values calculated in stage 3 of the procedure (boxes 1 and 2 in fig. 2). After that the delivery lot sizes are optimized using the threshold criteria of minimal difference between the total cost and the assumed storage cost in the given time period. Using this objective function allows for the minimal difference between expected and needed warehouse capacity.

In the last (sixth) stage of the procedure, the choice between all of the alternative solutions is made. The best choice is enabled by using the minimal total rent and transportation cost criteria for the given time period.

Therefore, the suggested procedure supposes the use of linear (boxes 1, 2 and 5 in fig, 2) and non-linear (boxes 3, 4 and 6 in fig, 2) optimization models. The models' structure allows for efficient implementation using the standard computing algorithms - simplex algorithm and evolutionary algorithm (genetic algorithms) - in common software, such as "Microsoft Excel".

\section{Conclusions and directions for future work}

This article contains a detailed procedure for determining the supply chain characteristics in industrial enterprises in a megapolis environment. As a result of the relevant works analysis it was concluded that the examined problem is highly relevant. The problem was posed and the corresponding main principles, input data and decision variables were determined. The procedure for solving the aforementioned problem was suggested, which is based on implementing a set of mathematical models including linear and non-linear optimization of delivery lot sizes and warehouse coordinates accordingly. These models can be efficiently used with standard computer software; however, they have the following demerits:

- when optimizing integer data of delivery lot sizes, it is possible that the strict correspondence between the real and provided demand volume proves impossible; in that case making an emergency supply or allowing stockouts should be taken into account and included in the threshold criteria and the objective function of the corresponding optimization model;

- no theoretical comparison of the sensitivity of transportation or rent cost to the warehouse coordinates is made in the optimization process; the analysis of said sensitivity would allow for determining the preferable order of optimization.

The follow-up research will allow eliminating the aforementioned demerits. It is planned to refine the procedure and subsequently put it into practice.

\section{References}

1. A. Diabat, J.-P. Richard, C.W. Codrington, Ann. Oper. Res., 203, 55-80 (2013)
2. A. Diabat, T. Aouam, L. Ozsen, IJADS, 2, 389-405 (2009)

3. S.M. Mousavi, V. Hajipour, S.T.A. Niaki, N. Alikar, Ap. Math. Mod., 37, 2241-2256 (2013)

4. S.M. Mousavi, S.T.A. Niaki, Appl. Math. Model, 37, 5109-5119 (2013)

5. S.M. Mousavi, S.T.A. Niaki, E. Mehdizadeh, M.R. Tavarroth, IJSS, 44, 1897-1912 (2013)

6. O.D. Palsule-Desai, Omega, 41, 780-796 (2013)

7. Y.-C. Tsao, G.-J. Sheen, Int. J. Prod. Econ., 135, 106-115 (2012)

8. J. Li, L. Liu, Int. J. Prod. Econ., 101, 89-98 (2006)

9. T.-Y. Lin, CIE, 66(1), 53-62 (2013)

10. K.-L. Huang, C.-W. Kuo, M.-L. Lu, Eur. J. Oper. Res., 234, 77-85 (2014)

11. Y.C. Chou, H.C. Yao, Y.S. Chun, Eur. J. Oper. Res., 189, 132-145 (2008)

12. T. Ozcan, N. Celebi, S. Esnaf, Expert Sys. Appl., 38, 9773-9779 (2011)

13. Y.C. Dong, H.J. Zhang, KBS, 58, $45-57$ (2014)

14. M. Ramezani, A.M. Kimiagari, B. Karimi, T.H. Hejazi, KBS, 59, 108-120 (2014)

15. Nia A. Roozbeh, Far M. Hemmati, S.T.A. Niaki, Int. J. Prod. Econ., 155, 259-171 (2013)

16. S.M. Mousavi, A. Bahreininejad, S.N. Musa, F. Yusof, J. Intell. Manuf., 1-16, 10.1007/s10845-0140970-z (2014)

17. S.M. Mousavi, N. Alikar, S.T.A. Niaki, A. Bahreininejad, CIE, 87, 543-560 (2015)

18. S. Karakaya, I.S. Bakal, CIE, 64, 696-707 (2013)

19. M.A. Rodriguez, A.R. Vecchietti, I. Harjunkoski, I.E. Grossmann, Comp. Chem. Eng., 62, 194-210 (2014)

20. Al-e-hashem S. Mirzapour, A. Baboli, Z. Sazvar, Eur. J. Oper. Res., 230, 26-41 (2013)

21. K. Subramanian, J.B. Rawlings, C.T. Maravelias, Comp. Chem. Eng., 64, 71-80 (2014)

22. S. Mondal, M. Maiti, CIE, 44. 105-117 (2002)

23. M.J. Liberatore, Oper. Res., 27, 391-396 (1979)

24. A.L. Guiffrida, Inventory Management: NonClassical Views (CRC Press, Taylor and Francis Group, FL, Boca Raton, 2009)

25. S.-C. Chang, J.-S. Yao, H.-M. Lee, Eur. J. of Oper. Res. 109, 183-202 (1998)

26. L.-Y. Ouyang, J.-S. Yao, Comput. Oper. Res. 29, 471-487 (2002)

27. N. Kazemi, E. Ehsani, M.Y. Jaber, IJAR, 51(8), $964-$ 972 (2010)

28. S. Bylka, Int. J. Prod. Econ., 143, 424-433 (2013)

29. A.E. Radaev, V.V. Kobzev, St. Peters, State Polytech. Univ. J. Econ., 233(6), 183-195 (2015)

30. A.E. Radaev, V.V. Kobzev, Cluster Economy and Industrial Policy: Theory and Instruments (Polytechnical University Publishing House, SaintPetersburg, 2015) 